\title{
Sudden onset of hind limb lameness in a 14-month-old beagle
}

On my weekly visit to a research laboratory, I encountered a 14-month-old dog with lameness in its left hind limb. The dog is one of a small colony of male beagles, aged between 1 and 3 years, used for pharmacodynamic and pharmacokinetic testing. The dogs are housed socially in small groups of four to eight beagles. Environmental conditions comply with the Guide for the Care and Use of Laboratory Animals.

The facility has a diverse environmental enrichment program for the beagles. The program involves provision of chew and tug toys, auditory (radio) and visual (children's programming) stimulation, and Mondayto-Friday group activity in a large open 'playroom' that contains soft children's play tunnels, ramps, benches, chew toys, and frisbees. One or two animal technicians directly supervise all environmental enrichment of the dogs. Usually four to six dogs play together in the playroom for 30-45 minutes. All dogs have the opportunity to play each weekday.

The 14-month-old dog in question developed the lameness in its left hind limb suddenly after an enrichment period in the playroom with three other dogs. The dog weighed $11.5 \mathrm{~kg}$ and was in good body condition (not obese). It had no previous history of illness, injury, or lameness.

Upon examining the dog on the same day that the lameness occurred, I found the dog

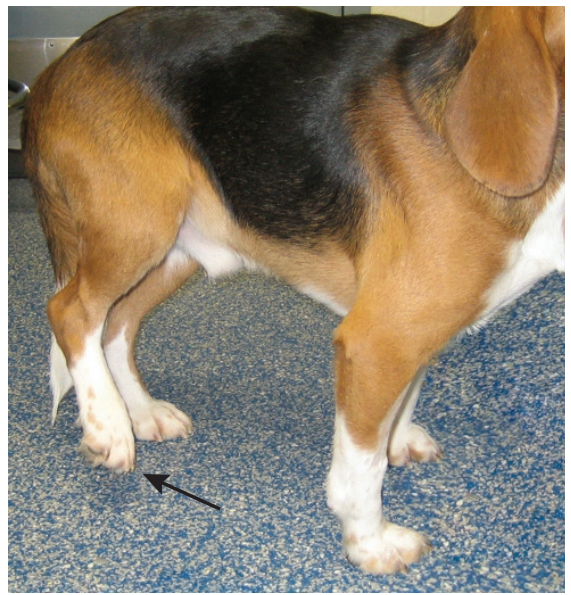

FIGURE 1 | A 14-month-old male beagle that displayed a sudden onset of lameness in the left hind limb. There is hind-limb instability characterized by the dog displaying almost no weight-bearing on the left hind leg. Notice how the left hind paw (arrow) is carried and not touching the floor. On clinical examination, the dog was bright, alert, and responsive, and no apparent abnormalities or pain could be detected.

bright, alert, and receptive. Body temperature, heart rate, respiratory rate, mucous membrane color, and capillary refill time were within normal limits. Examination of the lame left hind limb did not reveal any cuts or avulsed claws. Palpation of the hind leg and joints did not reveal any swelling, heat, or pain on manipulation. The dog was placed on the ground after examination and still displayed lameness, evinced by lifting the left hind leg off the ground (Fig. 1). The dog could walk but displayed almost no weight-bearing on the lame left hind leg.

The dog was placed in a dog run by itself and given meloxicam at a dose of $0.1 \mathrm{mg} / \mathrm{kg}$ by mouth every 24 hours for 4 days. Animal technicians examined the dog every day to see if there was any improvement. During this period, the dog showed no change in its personality or eating and drinking behavior. However, the dog continued to display hind-limb instability and lameness.

I examined the dog again on the fifth day after the lameness was first noticed. I paid special attention to the presence or absence of instability or pain in the left hind leg knee joint. With the dog laying down laterally on its side and restrained by a technician, I stabilized the position of the dog's left femur with one hand and attempted to move the dog's left tibia with the other hand. The tibia moved forward slightly.

Based on the history and clinical findings presented here, what do you suspect is the cause of the dog's lameness? How common is this condition in dogs? What is the treatment?

\section{What's your diagnosis?}

